\title{
Влияние случайных неоднородностей в пространственном распределении кластеров радиационных дефектов на перенос носителей заряда через тонкую базу гетеробиполярного транзистора при нейтронном воздействии
}

\author{
(C) А.С. Пузанов ${ }^{+}$, С.В. Оболенский ${ }^{+}$, В.А. Козлов ${ }^{+*}$ \\ + Нижегородский государственный университет им. Н.И. Лобачевского, \\ 603950 Нижний Новгород, Россия \\ * Институт ффизики микроструктур Российской академии наук, \\ 603950 Нижний Новгород, Россия \\ E-mail: obolensk@rf.unn.ru
}

(Получена 27 апреля 2016 г. Принята к печати 10 мая 2016 г.)

\begin{abstract}
Проведен анализ переноса электронов через тонкую базу гетеробиполярного транзистора на основе GaAs с учетом флуктуаций пространственного распределения кластеров радиационных дефектов при облучении потоком мгновенных нейтронов спектра деления. Теоретически показано, что однородное заполнение кластерами радиационных дефектов рабочей области приводит к минимальной деградации коэффициента усиления гетеробиполярного транзистора по постоянному току.
\end{abstract}

\section{1. Введение}

Взаимодействие быстрых нейтронов (с энергией в диапазоне 0.1-10 МэВ) с полупроводниковыми структурами приводит к тому, что частица выбивает из решетки один из атомов вещества [1], называемый первичным атомом отдачи. Этот атом с достаточно высокой энергией (до сотен кэВ), двигаясь в среде, сталкивается с атомом среды, в свою очередь выбивает его из занимаемого положения, и в результате каскад разветвляется наподобие дерева.

Нарушения кристаллической структуры, образующиеся вдоль траекторий движения атомов отдачи в виде точечных дефектов (вакансий и межузельных атомов), диффундируют, частично рекомбинируют, а частично образуют плотные скопления, называемые кластерами радиационных дефектов (КРД). Так как взаимодействие нейтронов с полупроводником, а также движение атомов отдачи и диффузия первичных точечных дефектов носят статистический характер, имеют место пространственные флуктуации концентрации КРД в облученном полупроводнике при макроскопически-однородном сечении упругого рассеяния нейтронов на ядрах атомов.

Кластер радиационных дефектов содержит набор глубоких уровней в запрещенной зоне полупроводника и является эффективным локальным центром рекомбинации носителей заряда, что оказывает существенное негативное влияние на функционирование биполярных транзисторов при нейтронном облучении. Уменьшение толщины базы транзистора снижает влияние увеличения темпа рекомбинации носителей заряда в ней при воздействии дефектообразующих излучений [2]. С другой стороны, в этом случае даже один КРД может эффективно перекрыть базу, создав диэлектрическое включение от эмиттера до коллектора. Таким образом, поражение субмикрометровых транзисторов носит ве- роятностный характер и определяется пространственным распределением кластеров радиационных дефектов, причем с уменьшением геометрических размеров биполярных транзисторов, прежде всего толщины базы, влияние данного эффекта усиливается. В пределе, повидимому, действие ионизирующих излучений на полупроводниковые приборы с размерами рабочей области, сравнимой с размерами КРД ( 10 нм при облучении $\mathrm{Si}$ и GaAs мгновенными нейтронами спектра деления), следует рассматривать как реализацию одиночных событий независимо от временны́х параметров воздействия (импульсного или статического).

Основным радиационно-критическим параметром биполярного транзистора является коэффициент усиления по постоянному току в схеме включения с общим эмиттером, $h_{21}$, изменение которого при нейтронном воздействии определяется выражением [3]

$$
\frac{1}{h_{21}^{n}}-\frac{1}{h_{21}^{0}}=k F_{n},
$$

где $h_{21}^{0}$ и $h_{21}^{n}-$ значения коэффициента усиления по постоянному току в схеме включения биполярного транзистора с общим эмиттером до и после облучения, соответственно, $k-$ коэффициент чувствительности биполярного транзистора к нейтронному воздействию, $F_{n}$ - флюенс мгновенных нейтронов спектра деления.

На макроскопическом уровне деградация коэффициента усиления по постоянному току в схеме включения с общим эмиттером при воздействии дефектообразующих излучений объясняется уменьшением времени жизни неосновных носителей заряда в базе биполярного транзистора в результате образования КРД. Типичное значение коэффициента чувствительности биполярного транзистора к нейтронному воздействию лежит в пределах $10^{-15}-10^{-16} \mathrm{~cm}^{2} /$ н, что обусловливает его радиационную стойкость порядка $10^{13}-10^{14} \mathrm{H} / \mathrm{cm}^{2}$. 
В работе [4] был проведен анализ переноса горячих электронов через тонкую базу биполярного транзистора при нейтронном воздействии в приближении квазиоднородного заполнения моделируемого объема КРД. Теоретически показано, что в определенном диапазоне концентраций КРД, соответствующих флюенсу нейтронов $\sim 10^{14} \mathrm{H} / \mathrm{cm}^{2}$, формируется потенциальный рельеф, уменьшающий среднее время пролета горячих электронов через базу. В результате на фоне общей деградации характеристик полупроводникового прибора по постоянному току может наблюдаться улучшение его высокочастотных характеристик, аналогично [5]. С дальнейшим ростом уровня облучения характерное расстояние между КРД становится сопоставимым с длиной волны де Бройля [6] и база транзистора разбивается на сетку нерегулярным образом связанных каналов, похожих по своим свойствам на короткие квантовые нити, образуемые сквозными квантово-размерными соответствиями.

Данная работа посвящена детальному анализу влияния флуктуаций концентрации КРД на перенос носителей заряда через тонкую базу с учетом вышеописанных эффектов. Решение данной проблемы позволит объективно оценивать радиационную стойкость СВЧ и КВЧ гетеробиполярных транзисторов, а также учитывать указанные эффекты при разработке перспективной элементной базы.

\section{2. Математическая модель}

В рамках данной работы последовательно рассчитывали реализацию распределения КРД в моделируемом объеме, а затем перенос носителей заряда (электронов) через тонкую базу гетеробиполярного транзистора на основе GaAs.

\section{1. Расчет пространственного распределения кластеров радиационных дефектов}

Для расчета распределения КРД в полупроводнике при воздействии мгновенных нейтронов спектра деления рассматривался процесс движения первичного атома отдачи, получающего энергию при столкновении с нейтроном. Для моделирования движения первичного атома отдачи в веществе использовалась программа, аналогичная известной программе SRIM, алгоритм которой описан в работе [7]. Траектория каждого первичного атома отдачи начиналась с введения его положения (равномерно распределенного для моделируемого объема), направления движения (изотропного в полупространстве) и энергии, выбираемой равновероятно из диапазона от 0 до $E_{\max }[8]$, где

$$
E_{\max }=\frac{4 A}{(1+A)^{2}} E_{n}
$$

Здесь $A-$ атомный вес. Энергетический спектр мгновенных нейтронов аппроксимировали зависимо- стью $[9,10]$

$$
f\left(E_{n}\right) \propto \exp \left(-E_{n}\right) \sinh \left(\sqrt{2 E_{n}}\right),
$$

где $E_{n}-$ энергия нейтрона в МэВ.

Затем прослеживалась последовательность столкновений первичного атома отдачи с атомами вещества, а между столкновениями свободный пробег первичного атома отдачи предполагался прямолинейным. На пути каждого свободного пробега энергия атома отдачи уменьшалась на величину электронных потерь энергии, а после столкновения - на величину потерь, связанную с излучением фононов. Если атом материала получал энергию, которая превышает пороговое значение

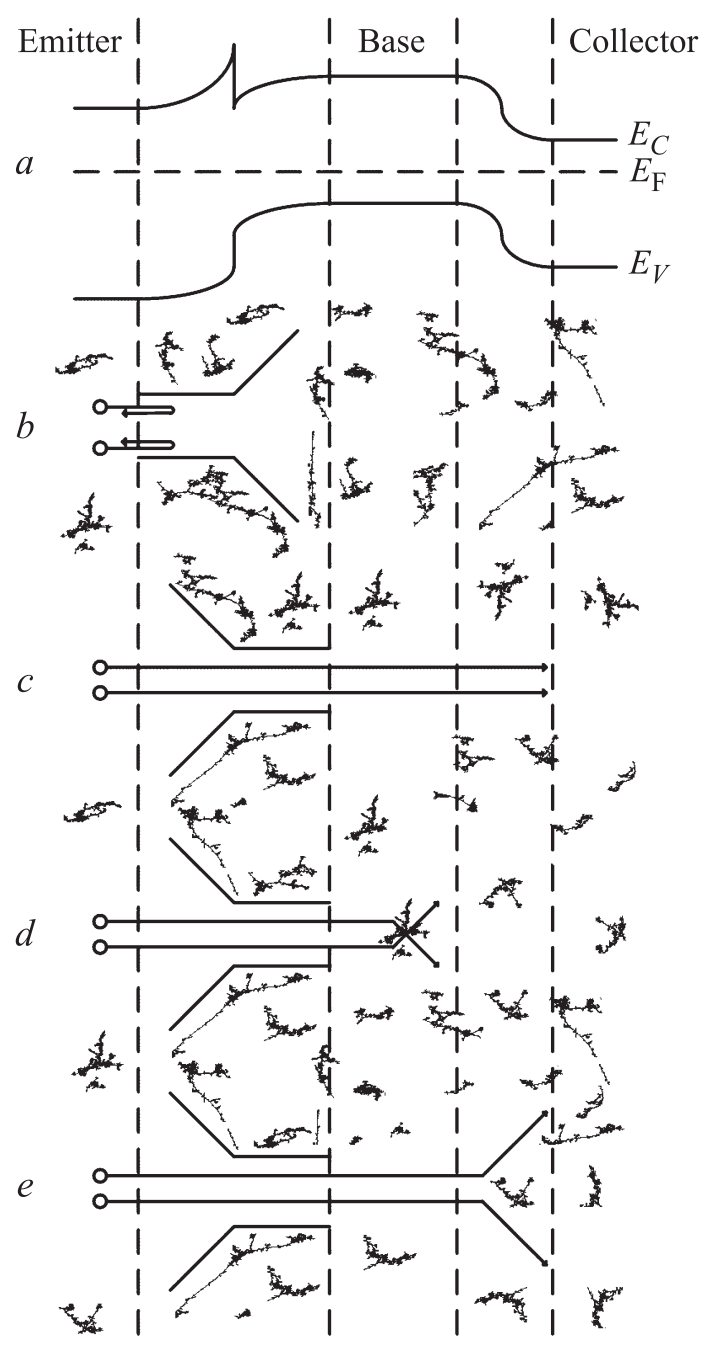

Рис. 1. Модельные пространственные распределения КРД: $a$ - зонная структура гетеробиполярного транзистора на основе GaAs; $b$ - конфигурация „обратный рупор“; $c$ конфигурация „прямой рупор“; $d-$ конфигурация „прямой рупор“ с кластером радиационных дефектов в канале базы; $e-$ конфигурация „прямой рупор“ с кластером радиационных дефектов на выходе из канала. Показаны электроны в зоне проводимости $E_{C}$ (кружки). Конфигурация КРД рассчитана методом Монте-Карло. 
Таблица 1. Параметры зонной структуры GaAs

\begin{tabular}{l|c}
\hline \multicolumn{1}{c}{ Параметр } & Значение параметра \\
\hline Эффективная масса электрона в Г-долине $m_{\Gamma} / m_{0}$ & 0.063 \\
Продольная эффективная масса электрона в $L$-долине $m_{L, l} / m_{0}$ & 1.538 \\
Поперечная эффективная масса электрона в $L$-долине $m_{L, t} / m_{0}$ & 0.127 \\
Продольная эффективная масса электрона в $X$-долине $m_{X, l} / m_{0}$ & 1.987 \\
Поперечная эффективная масса электрона в $X$-долине $m_{X, t} / m_{0}$ & 0.229 \\
Энергетический зазор между долинами $\Gamma-L$, эВ & 0.323 \\
Энергетический зазор между долинами $\Gamma-X$, эВ & 0.457 \\
Константа непараболичности в Г-долине $\alpha$,эВ & 0.620 \\
Константа непараболичности в $L$-долине $\alpha$,эВ & 0.500 \\
Константа непараболичности в $X$-долине $\alpha$, эВ & 0.300 \\
Эффективная масса легких дырок $m_{l h} / m_{0}$ & 0.082 \\
Эффективная масса тяжелых дырок $m_{h h} / m_{0}$ & 0.450 \\
Ширина запрещенной зоны, эВ & 1.424
\end{tabular}

Примечание. $m_{0}$ - масса свободного электрона.

энергии образования элементарных точечных дефектов, его поведение прослеживалось таким же образом, как и поведение налетающего первичного атома отдачи. Траектория первичного атома отдачи обрывалась при уменьшении его энергии до пороговой энергии образования элементарных точечных дефектов. Пример расчета пространственного распределения КРД в объеме гетеробиполярного транзистора с тонкой базой на основе GaAs приведен на рис. 1.

\section{2. Расчет переноса носителей заряда через тонкую базу биполярного транзистора при нейтронном воздействии}

Анализ переноса носителей заряда через тонкую базу биполярного транзистора при нейтронном воздействии проведен при помощи самосогласованного решения кинетического уравнения Больцмана и уравнения Пуассона. Типичные модельные пространственные распределения КРД приведены на рис. 1. При расчете учитывались флуктуации пространственного распределения КРД в эмиттерном переходе, инжектирующем в базу электроны, приводящие как к „закупориванию“ проводящего канала - при конфигурации „обратный рупор“ (рис. 1, b), так и облегчающие ввод электронов в канал - „прямой рупор“ (рис. 1,c) [4]. Метод расчета ввода электронов в канал адаптирован при использовании модели работы [11]. В результате пространственное распределение КРД в эмиттерном переходе биполярного транзистора формирует для электронов, инжектируемых в базу, распределение по энергии и диаграмму направленности.

Пространственное распределение КРД в базе и коллекторном переходе также влияет на прохождение носителей заряда. Так, формирование КРД внутри (рис. 1, $d$ ) и на выходе из проводящего канала (рис. $1, e)$ существенно уменьшает коэффициент прохождения электронов, причем в зависимости от энергии электронов процесс носит резонансный характер, аналогично результатам моделирования влияния интерференции на самосогласованном потенциале и случайных заряженных центрах в квантовом канале полевого транзистора из обзора [12].

Алгоритм Монте-Карло в отсутствие облучения с учетом самосогласованного решения уравнения Пуассона взят из работы [13], однако в данной работе зонная структура GaAs задавалась аналитически с параметрами, приведенными в табл.1.

На каждом шаге алгоритма для каждой из модельных частиц рассчитывалось время свободного пробега $[14,15]$

$$
\tau=\frac{1}{\Gamma} \ln (\mathbf{r})
$$

где $\mathbf{r}$ - случайное число равномерного распределения в интервале от 0 до 1 , Г - суммарная частота рассеяния, которая в данной работе полагалась равной $4 \cdot 10^{14} \mathrm{c}^{-1}$. Движение модельных частиц в координатно-импульсном $(r, p)$ 6-мерном фазовом пространстве между столкновениями описывалось уравнениями [13]

$$
\begin{gathered}
\frac{d r}{d t}=\nabla_{p} E(p), \\
\frac{d p}{d t}=\mp q \nabla_{r} \varphi(r),
\end{gathered}
$$

где $E(p)$ - зависимость энергии модельной частицы от импульса, $\varphi(r)$ - пространственное распределение потенциала электрического поля, $t-$ время, $q-$ заряд электрона. Знак „ $+{ }^{“}$ в уравнении (6) относится к электронам, „-““- - д дыркам.

После свободного пробега выбор механизма рассеяния проводится с помощью случайного числа $\mathbf{r}$ из равномерного распределения в интервале от 0 до 1 . Процесс рассеяния с номером $m$ выбирается, если [15]

$$
\frac{1}{\Gamma} \sum_{i=1}^{m-1} \lambda_{i}(E)<\mathbf{r}<\sum_{i=1}^{m} \lambda_{i}(E),
$$

где $\lambda(E)$ - зависимость частоты рассеяния от энергии модельной частицы. 
Таблица 2. Энергии $E_{a}$ (относительно зоны проводимости) и сечения захвата электронов $\sigma_{h}$ в облученном $n$-GaAs $[19,20]$

\begin{tabular}{c|c|c}
\hline Ловушка & $\begin{array}{c}\text { Энергия активации } \\
\text { эмиссии } E_{a},{ }^{\mathrm{B}}\end{array}$ & $\begin{array}{c}\text { Сечение } \\
\text { захвата } \sigma_{n}, \mathrm{~cm}^{2}\end{array}$ \\
\hline$E 1$ & 0.045 & $2 \cdot 10^{-15}$ \\
$E 2$ & 0.140 & $1 \cdot 10^{-13}$ \\
$E 3$ & 0.300 & $6 \cdot 10^{-15}$ \\
$E 4$ & 0.760 & $3 \cdot 10^{-14}$ \\
$E 5$ & 0.960 & $2 \cdot 10^{-12}$
\end{tabular}

Таблица 3. Энергии $E_{a}$ (относительно валентной зоны) и сечения захвата дырок $\sigma_{p}$ в облученном $p$-GaAs $[19,20]$

\begin{tabular}{c|c|c}
\hline Ловушка & $\begin{array}{c}\text { Энергия активации } \\
\text { эмиссии } E_{a}, \text { эВ }\end{array}$ & $\begin{array}{c}\text { Сечение } \\
\text { захвата } \sigma_{p}, \mathrm{~cm}^{2}\end{array}$ \\
\hline H0 & 0.06 & $2 \cdot 10^{-16}$ \\
H1 & 0.25 & $1 \cdot 10^{-15}$ \\
H2 & 0.42 & - \\
H3 & 0.54 & $7 \cdot 10^{-16}$ \\
H4 & 0.79 & $7 \cdot 10^{-14}$ \\
H5 & 0.85 & $9 \cdot 10^{-15}$
\end{tabular}

Как отмечается в монографии [15], наиболее важными механизмами рассеяния в $\mathrm{GaAs}$ являются рассеяние на полярных оптических фононах, на ионизованных примесях, пьезоэлектрическое и акустическое рассеяние, а также междолинное рассеяние в сильном электрическом поле. Частоты и угловые параметры рассеяния, используемые в данной работе, взяты из указанной монографии.

Согласно основополагающей работе [16], КРД представляют собой заряженные включения сферической или эллипсоидальной формы, свойства которых отличаются от свойств окружающего их неповрежденного полупроводника. В центре КРД находится обогащенное дивакансиями ядро, которое окружено тонкой по сравнению с его размерами оболочкой, состоящей из комплексов вакансий с атомами примеси. В широкозонных полупроводниках, в частности в GaAs, ядро КРД по положению уровня Ферми $F_{\mp}$ аналогично локальной области нелегированного материала $E_{\mathrm{F}}=E_{V}+0.6$ э [17]. Таким образом, КРД представляет собой потенциальный барьер для основных и яму для неосновных носителей заряда. Радиус ядра КРД рассчитывался согласно подходу, предложенному в работе [18], а радиус окружающей его области пространственного заряда варьировался в зависимости от концентрации легирующей примеси и учитывался в самосогласованном распределении потенциала электрического поля.

Энергетическая структура уровней дефектов, образованных КРД, и сечения захвата подвижных носителей заряда на них взяты из работ $[19,20]$ и приведены в табл. 2,3 для $n$-GaAs и $p$-GaAs соответственно. Также следует отметить имеющий место значительный разброс экспериментальных данных, обусловленный, по-видимому, различными условиями облучения материала, исходной концентрацией дефектов, примесей и т.д.

Рекомбинация носителей заряда рассматривалась как один из вариантов развития событий, определяемых выражением (7). При этом модельная частица, для которой реализовалось событие рекомбинации, исключалась из дальнейшего рассмотрения вместе с наиболее близко расположенной модельной частицей противоположного заряда. Частота процесса рекомбинации определялась по формуле, аналогичной приведенной в [21]:

$$
\lambda_{\mathrm{rec}}=v N_{\mathrm{cl}} \sum_{i} \sigma_{i},
$$

где $v$ - скорость модельной частицы, $\sigma_{i}-$ сечения захвата электронов и дырок, $N_{\mathrm{cl}}$ - концентрация КРД.

\section{3. Результаты и обсуждение}

Как показано на рис. 1, распределение КРД в структуре транзистора носит резко неоднородный характер. В табл. 4 приведены характерные параметры КРД в объемном GaAs, использовавшиеся в расчетах переноса носителей заряда через тонкую базу.

Для модельных пространственных распределений КРД, приведенных на рис. 1, рассчитана средняя направленная скорость движения электронов от эмиттера к коллектору (рис. 2). Толщина базы принималась равной 300 нм. Как обсуждалось в работе [4], конфигурация

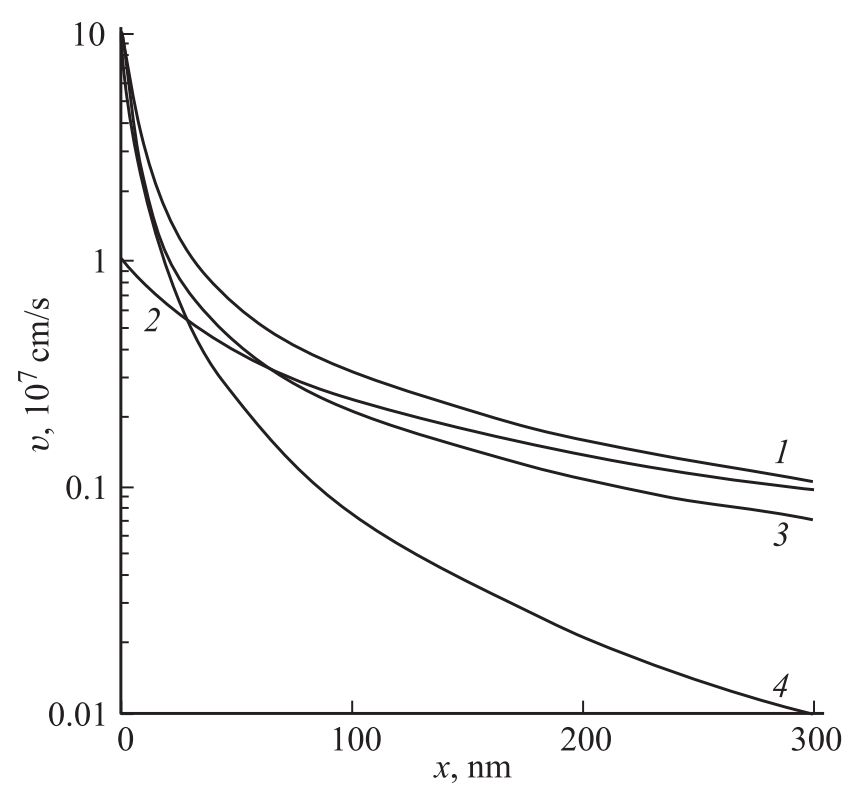

Рис. 2. Зависимость направленной скорости $v$ от координаты $x$ в базе гетеробиполярного транзистора на основе GaAs: 1 - конфигурация КРД „прямой рупор“; 2 - до облучения; 3 - конфигурация КРД „прямой рупор“ с КРД внутри канала в базе; 4 - конфигурация КРД „прямой рупор“ с КРД на выходе из канала в коллекторном переходе. 
Таблица 4. Параметры КРД в объемном GaAs

\begin{tabular}{|c|c|c|c|c|c|}
\hline \multicolumn{2}{|c|}{ Флюенс нейтронов, н/см² } & $10^{12}$ & $10^{13}$ & $10^{14}$ & $10^{15}$ \\
\hline \multicolumn{2}{|c|}{ Концентрация КРД, см${ }^{-3}$} & $2 \cdot 10^{11}$ & $2 \cdot 10^{12}$ & $2 \cdot 10^{13}$ & $2 \cdot 10^{14}$ \\
\hline \multicolumn{2}{|c|}{ Среднее расстояние между центрами КРД, нм } & 1710 & 794 & 368 & 171 \\
\hline $\begin{array}{c}\text { Концентрация } \\
\text { легирующей } \\
\text { примеси, см }{ }^{-3}\end{array}$ & $\begin{array}{c}\text { Область } \\
\text { пространственного } \\
\text { заряда КРД, нм }\end{array}$ & \multicolumn{4}{|c|}{$\begin{array}{c}\text { Среднее расстояние между границами областей } \\
\text { пространственного заряда КРД, нм }\end{array}$} \\
\hline $\begin{array}{l}2.4 \cdot 10^{15} \\
6.8 \cdot 10^{15} \\
1.3 \cdot 10^{16} \\
3.0 \cdot 10^{16} \\
1.0 \cdot 10^{17} \\
5.0 \cdot 10^{17}\end{array}$ & $\begin{array}{r}171 \\
119 \\
94 \\
71 \\
46 \\
27\end{array}$ & $\begin{array}{l}1539 \\
1591 \\
1616 \\
1639 \\
1663 \\
1683\end{array}$ & $\begin{array}{l}623 \\
625 \\
700 \\
723 \\
747 \\
767\end{array}$ & $\begin{array}{l}197 \\
250 \\
274 \\
298 \\
322 \\
342\end{array}$ & $\begin{array}{r}0 \\
52 \\
77 \\
100 \\
125 \\
144\end{array}$ \\
\hline
\end{tabular}

КРД типа „прямой рупор“ способна улучшить характеристики гетеробиполярного транзистора, в частности уменьшить время пролета через базу. При этом, однако, попадание КРД внутрь или на выход из канала приводит к интенсивному рассеянию электронов, причем, если в базе КРД является потенциальной ямой для электронов, что приводит к преимущественно малоугловому рассеянию и относительно слабому уменьшению направленной скорости, то в коллекторном переходе КРД является потенциальным барьером и рассеяние электронов происходит в том числе и назад, что существенно снижает величину направленной скорости в базе гетеробиполярного транзистора.

Выходные вольт-амперные характеристики (ток коллектора $I_{c}-$ напряжение коллектор-база $\left.U_{c b}\right)$ гетеробиполярного транзистора с базой на основе GaAs толщиной 300 нм приведены на рис. 3. Увеличение направленной составляющей скорости электронов в базе, вызванное формированием более „острой“ диаграммы направленности по скоростям электронов по направлению нормали к границе гетероперехода эмиттер-база в конфигурации КРД типа „прямой рупор“, дает рост тока коллектора. Такое поведение гетебиполярного транзистора при нейтронном облучении является аномальным и приводит к улучшению его характеристик. При этом попадание КРД внутрь или на выход из канала приводит к практически идентичному ухудшению характеристик гетеробиполярного транзистора. Причиной этого является то обстоятельство, что, несмотря на малоугловой характер рассеяния электронов в базе, приводящий к сохранению значительной части направленной скорости, некоторая их часть захватывается КРД с последующей рекомбинацией, что приводит к снижению тока коллектора. Также отметим, что несмотря на возможность локального улучшения характеристик гетеробилярного транзистора при нейтронном облучении, вероятность поражения КРД области эмиттера, базы, коллектора, образования конфигурации типа „обратный рупор“ в эмиттерном переходе значительно выше, чем вероят- ность образования конфигурации типа „прямой рупор“. Поэтому в подавляющем большинстве гетеробиполярных транзисторов характеристики будут ухудшаться при нейтронном воздействии. Этот тезис качественно подтверждают экспериментальные результаты работ $[5,6]$.

Зависимость относительной деградации коэффициента усиления по постоянному току биполярного транзистора с тонкой базой от флюенса нейтронного воздействия для однородного и неоднородного заполнения КРД рабочего объема биполярного транзистора приведена на рис. 4.

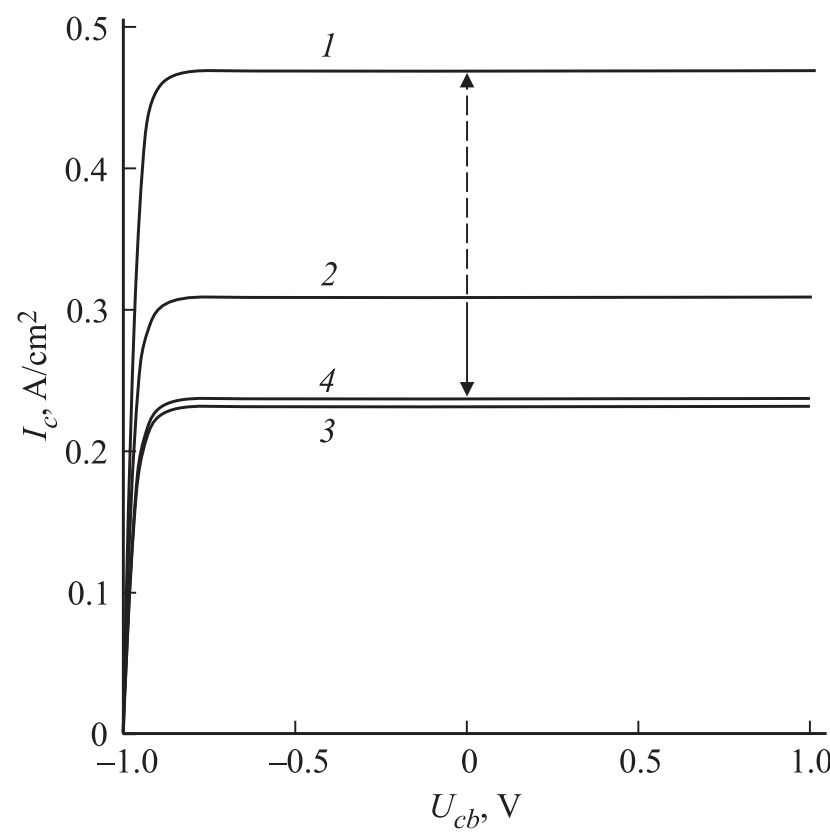

Рис. 3. Выходная вольт-амперная характеристика $I_{c}\left(U_{c b}\right)$ гетеробиполярного транзистора с тонкой базой на основе GaAs: 1 - конфигурация КРД „прямой рупор“; 2 - до облучения; 3 - конфигурация КРД „прямой рупор“ с КРД внутри канала в базе; 4 - конфигурация КРД „прямой рупор“ с КРД на выходе из канала в коллекторном переходе. Стрелками отмечены направления нормальной $(\downarrow)$ и аномальной $(\uparrow)$ деградации характеристик 


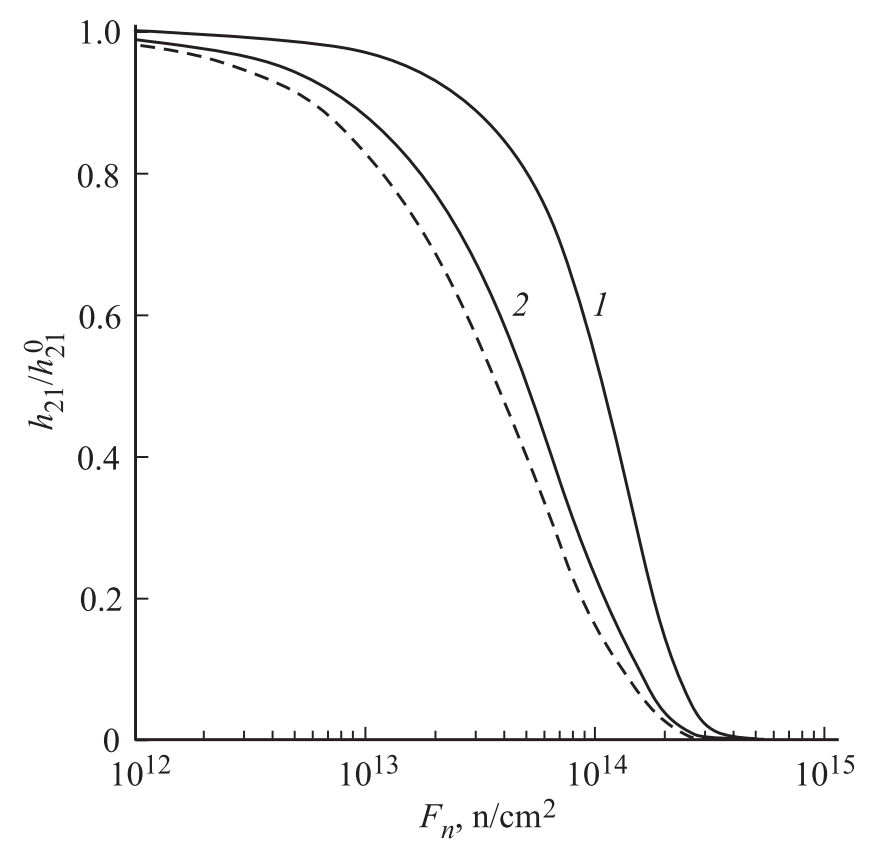

Рис. 4. Относительная деградация коэффициента усиления по постоянному току биполярного транзистора с тонкой базой в зависимости от флюенса нейтронного воздействия для однородного (сплошная линия) и неоднородного (штриховая) заполнения кластерами радиационных дефектов рабочего объема: толщина базы 30 (1) и 300 нм (2). Для неоднородного заполнения кластерами радиационных дефектов приведен наихудший случай.

Установлено, что однородное заполнение КРД рабочего объема вызывает минимальную деградацию коэффициента усиления по постоянному току биполярного транзистора с тонкой базой, так как во всех вычислительных экспериментах по случайному заполнению КРД рабочего объема гетеробиполярного транзистора в нем реализовывались негативные конфигурации КРД. Последнее важно при проведении расчетных оценок радиационной стойкости субмикрометровых полупроводниковых приборов и интерпретации результатов облучательных экспериментов. Деградация коэффициента усиления по постоянному току биполярного транзистора при неоднородном заполнении рабочего объема КРД практически не зависит от толщины базы в диапазоне 30-300 нм.

\section{4. Заключение}

Результаты проведенных расчетов показывают, что учет флуктуаций пространственного распределения КРД, возникающих в субмикрометровых полупроводниковых приборах при воздействии потока нейтронов спектра деления, позволяет интерпретировать ряд явлений, в частности „внезапный“ отказ или скачкообразное ухудшение параметров транзисторов в облучательных экспериментах. Подобное поведение, скорее, характерно для воздействия тяжелых заряженных частиц космического пространства, что указывает на общность процессов, протекающих в субмикрометровых полупроводниковых приборах при воздействии различных видов проникающих излучений, и на необходимость разработки детальной методологии расчетной оценки радиационной стойкости изделий микроэлектроники, в том числе при помощи физико-топологического моделирования, на основе вероятностного подхода.

Работа выполнена при поддержке программ РАН и программы повышения конкурентоспособности ННГУ им. Н.И. Лобачевского среди ведущих мировых научно-образовательных центров. Работа выполнена при поддержке грантами РФФИ № 14-02-0058, 15-02-07935, МОН РФ (соглашение от 27.09.2013 г. № 02.В.49.21.003) и Государственным заданием $\mathrm{MOH}$ РФ (код проекта 2183).

\section{Список литературы}

[1] И.М. Дремин, О.В. Иванов, В.А. Нечитайло, Н.М. Соболевский, А.В. Субботин, В.П. Шевелько. ЖЭТФ, 125 (2), 362 (2004).

[2] В.К. Киселев, С.В. Оболенский, А.С. Пузанов. Вестн. ННГУ, № 2 (1), 56 (2013).

[3] В.Н. Афанасьев, В.Б. Бычков, А.В. Кедров, А.В. Лыжин, О.А. Мингазов, В.П. Пудов, А.К. Серебряков. Вопросы атомной науки и техники. Серия: Физика радиационного воздействия на радиоэлектронную аппаратуру. № 3-4, 119 (2001).

[4] А.С. Пузанов, С.В. Оболенский, В.А. Козлов. ФТП, 49 (1), 71 (2015).

[5] С.В. Оболенский. Микроэлектроника, 33 (2), 153 (2004).

[6] В.Т. Громов, М.А. Китаев, Е.В. Киселева, В.А. Козлов, С.В. Оболенский, В.П. Шукайло. Микроэлектроника, 34 (6), 424 (2005).

[7] J.P. Biersak et al. Nucl. Instrum. Meth., 174, 257 (1980).

[8] Е.Г. Григорьев, Ю.А. Перлович, Г.И. Соловьев, А.Л. Удовский, В.Л. Якушин. Физическое материаловедение. Том 4. Физические основы прочности. Радиачионная физика твердого тела. Компьютерное моделирование (М., МИФИ, 2008).

[9] Г.Г. Бартоломей, В.Д. Байбаков, М.С. Алтухов, Г.А. Бать. Основы теории и методы расчета ядерных энергетических реакторов (М., Энергоатомиздат, 1982).

[10] А.Н. Климов. Ядерная физика и ядерные реакторы (М., Энергоатомиздат, 1985).

[11] В.А. Козлов, С.В. Оболенский, В.Б. Шмагин, З.Ф. Красильник. ФТП, 46 (1), 134 (2012).

[12] К.А. Валиев, В.В. Вьюрков, А.А. Орликовский. Успехи соврем. радиоэлектроники, № 6,7 (2010).

[13] M.V. Fischetti, S.E. Laux. Phys. Rev. B, 38 (14), 9721 (1988).

[14] C. Jacoboni, L. Reggiani. Rev. Mod. Phys., 55 (3), 645 (1983).

[15] М. Шур. Современные приборы на основе арсенида галлия (М., Мир, 1991).

[16] B.R. Gossick. J. Appl. Rhys., 30 (8), 1214 (1959).

[17] V.N. Brudnyi, S.N. Grinyaev, V.E. Stepanov. Physica B: Condens. Matter, 212 (4), 429 (1995).

[18] А.С. Пузанов, С.В. Оболенский, В.А. Козлов, Е.В. Волкова, Д.Г. Павельев. ФТП, 49 (12), 1585 (2015). 
[19] D. Pons, J.C. Bourgoin. J. Phys. C: Sol. St. Phys., 18 (20), 3839 (1985).

[20] В.А. Козлов, В.В. Козловский. ФТП, 35 (7), 769 (2001).

[21] П.Т. Орешкин. Физика полупроводников и диэлектриков (М., Высш. шк., 1977).

Редактор Л.В. Шаронова

\section{Influence of random inhomogeneities in the spatial distribution of radiation defect clusters on charge carriers transport through the heterojunction bipolar transistor thin base at neutron irradiation}

A.S. Puzanov*, S.V. Obolenskiy*, V.A. Kozlov ${ }^{+*}$

+ Lobachevskii University of Nizhny Novgorod, 603950 Nizhny Novgorod, Russia

* Institute for Physics of Microstructures,

Russian Academy of Sciences,

603950 Nizhny Novgorod, Russia

Abstract Analysis of electron transport through the thin base of GaAs heterojunction bipolar transistor, taking into account fluctuations in the spatial distribution of clusters of radiation defects upon neutron irradiation has been carried out. It is shown theoretically that the homogeneous filling of clusters of radiation defects workspace leads to a minimum of degradation of the heterojunction bipolar transistor gain. 